\title{
Hierarchically porous composites fabricated by hydrogel templating and viscous trapping techniques
}

Benjamin R. Thompson, ${ }^{a}$ Tommy S. Horozov, ${ }^{\text {a }}$ Simeon D. Stoyanov ${ }^{\text {b,c,d }}$ and Vesselin N. Paunov*a

a School of Mathematics and Physical Sciences (Chemistry), University of Hull, Hull, United Kingdom;

${ }^{b}$ Unilever R\&D Vlaardingen, Olivier van Noortlaan 120, 3133 AT Vlaardingen, the Netherlands;

${ }^{c}$ Laboratory of Physical Chemistry and Soft Matter, Wageningen University, $6703 \mathrm{HB}$ Wageningen, The Netherlands;

${ }^{d}$ Department of Mechanical Engineering, University College London, Torrington Place, London WC1E 7JE, UK.

$$
\begin{gathered}
* \text { Corresponding author contact details: } \\
\text { Phone: }+44(0) 1482 \text { 465660, Fax: }+44(0) 1482466410 \text {; } \\
\text { Email: v.n.paunov@hull.ac.uk }
\end{gathered}
$$

Submitted to Materials \& Design (Elsevier) 


\begin{abstract}
Two methods for the preparation of hierarchically porous composites have been developed and explored. The first involved templating mixed slurries of hydrogel beads with two different average bead size distributions with gypsum slurry which allows for precise control over the porosity, pore size distributions and hierarchical microstructure of the hardened composite after the evaporation of the water from the hydrogel beads. The other technique utilised the viscosity of methylcellulose solution to suspend gypsum particles as they form an interlocked network. By varying the volume percentage of methylcellulose solution used, it is possible to control the porosity of the dried sample. The mechanical and thermal insulation properties of the composites as a function of both their porosity and pore size were investigated. Both methods demonstrate an inexpensive approach for introducing porosity in gypsum composites which reduces their thermal conductivity, improves their insulation properties and allows economic use of the matrix material whilst controlling their mechanical properties. Such composites allow for tuneable porosity without significantly compromising their strength which could find applications in the building industry as well as structuring of other composites for a variety of consumer products.
\end{abstract}

KEYWORDS: hydrogels, porous materials, methylcellulose, agar, hydrogel templating, viscous trapping, thermal conductivity, compressional strength, hierarchical porosity 


\section{Introduction}

Gypsum based composites are commonly used in buildings as dry walls or ceilings due to their relatively low cost, low thermal conductivity and passive fire resistance. This fire resistance stems from a large increase in specific heat during the temperature driven removal of the water of crystallisation[1,2]. One way to further improve the thermal insulating properties of gypsum and to decrease its thermal conductivity, requires pores to be incorporated within the gypsum network. Air has a significantly lower thermal conductivity than a solid phase, therefore incorporating porosity within a material decreases its thermal conductivity. Heat transfer in a porous material is accomplished through a combination of lattice vibrations in the solid phase, conduction through collisions of gas molecules within the pores, through thermal radiation and, if the pore sizes are sufficiently large, convection within the pores[3].

Controlling the thermal and mechanical properties of porous and composite materials is an area of significant interest[4-6]. One group has demonstrated how the inclusion of graphene into a polymeric aerogel can produce materials where the thermal conductivity and compressive stress can be increased by increasing the amount of graphene incorporated within the composite[7]. The same effect has been shown when preparing a composite foam of carbon with graphite filler[8]. Another group has prepared a hierarchically porous composite of carbon with silica nanoparticles incorporated within. They achieved thermal conductivities $98 \%$ lower than the non-porous carbon which they attributed to the presence of a mesoporous structure[9].

This leads to the Knudsen effect of decreasing the thermal conductivity of air confined in pores smaller than its mean free path. The presence of the silica nanoparticles also contributed to reducing the thermal conductivity of the composites due to them having a lower thermal conductivity than carbon. Therefore the heat flow through the material avoids these regions thus decreasing the phonon mean free path and increasing phonon scattering[10].

Recently, it has been demonstrated that a cheap, easy and environmentally friendly method of introducing porosity into a material, that also gives a large amount of control over both the porosity and pore size, is using hydrogel bead templates. A hydrogel bead templating technique to produce porous materials has previously been reported that involved the use of gellan or polyacrylamide hydrogel beads as templates to introduce porosity into a variety of materials[11]. By combining slurries of the matrix material and hydrogel beads in controlled volume ratios, followed by subsequent curing and then drying, porous materials were obtained with a porosity controlled by the volume of hydrogel beads used. Furthermore, the average pore size of the composite was determined by the size of the hydrogel beads used. 
Here, this method has been extended further to make hierarchically porous gypsum composites by using agar hydrogel beads of different sizes as the templates. The use of agar hydrogel instead of gellan or polyacrylamide is due to the gelling fraction of agar, agarose, being a nonionic hydrocolloid that does not interact with calcium ions from the gypsum slurry which allows for a better control during the formulation of these composites[12]. Fig. 1A shows schematically the process of hydrogel beads templating for fabrication of porous gypsum composites.

A complementary viscous trapping method for controlling the porosity of gypsum through the use of methylcellulose (MC) solution has also been developed. Mixing gypsum slurry with a viscous MC solution during the gypsum setting process, stops the sedimentation of the gypsum particles and allows more time for them to hydrate and interconnect into a continuous network. This method allows control over the porosity, but the pore size increases with increasing volume of MC solution used due to it essentially expanding the innate porosity of gypsum. Schematics of the viscous trapping method for introducing porosity in gypsum composites is presented in Fig. 1B. Both methods can also be used to introduce hierarchical porosity in cement, ceramics, food, home and personal care products and other composite materials of similar setting process. In the current paper, an investigation into how the thermal conductivity and mechanical properties of the porous gypsum composites vary with porosity and pore size has been performed.

This paper is organised as follows: Section 2 describes the methods and materials used to prepare porous and hierarchically porous gypsum composites using the hydrogel bead templating technique and the viscous trapping method. In Section 3 the results on the measurement of the composite thermal conductivity and their mechanical properties as a function of porosity and pore size are presented. Furthermore, the microstructure has been investigated in detail in this section.

\section{Experimental}

\subsection{Materials}

Agar was purchased from Special Ingredients and MC (A4M, food grade) was a gift from The Dow Chemical Company, Germany. Gypsum powder $\left(\mathrm{CaSO}_{4} \cdot 0.5 \mathrm{H}_{2} \mathrm{O},<3 \%\right.$ crystalline silica impurities, Lafarge Prestia) was purchased from Fred Aldous: Art, design and craft supplies. 
Deionised water was obtained by a Milli-Q purification system (Millipore) and used in all experiments.

\subsection{Preparation of hydrogel beads and MC solution}

Agar hydrogel $(2.0 \% \mathrm{w} / \mathrm{v})$ was prepared by heating water to $97^{\circ} \mathrm{C}$ using a water bath, adding the appropriate mass of agar powder and homogenising with an Ultra-Turrax homogeniser for 15 minutes whilst being covered with foil to minimise evaporation of water. It was allowed to set at room temperature and then left in a fridge $\left(4^{\circ} \mathrm{C}\right)$ for 24 hours before use. The resulting hydrogel was transferred to a Tefal food processor minipro food blender (a $500 \mathrm{~W}$ blender with 3 stacked blades) and blended at full power for either 10 seconds to produce 'large beads' or for 600 seconds to produce 'small beads'. MC solution $(0.5 \% \mathrm{w} / \mathrm{v})$ was prepared via addition of the appropriate mass of MC powder to cold water whilst homogenising. It was then placed in a fridge overnight at $4{ }^{\circ} \mathrm{C}$ to ensure complete dissolution of the MC. Prior to use, it was removed from the fridge and brought to room temperature.

\subsection{Fabrication of porous gypsum composites}

Gypsum powder was mixed with water in the mass ratio 1.45:1 (gypsum : water) to produce a thick aqueous slurry of gypsum. This slurry was mixed with either small agar beads, large agar beads or MC aqueous solution. Four different volume percentages of slurry of small or large hydrogel beads or MC solution were used during preparation (15\%, 30\%, 45\% and 60\%) as well as a control sample of gypsum alone. These composite slurries were poured into a cylindrical mould (inner dimensions were $60 \mathrm{~mm}$ height and $60 \mathrm{~mm}$ diameter) and a lid was placed on top. The lid had several vertical metal cylinders attached to produce holes in the composite samples at the locations for the cartridge heater and thermocouples to fit. This was done to ensure that the placement of the heater and thermocouples would be the same in each sample. After pouring into the mould and placing the lid on, the samples were allowed to cure at room temperature for 1 hour. Subsequently, the lid was removed and they were transferred to an oven $\left(40^{\circ} \mathrm{C}\right)$ to dry until reaching a constant mass. Schematics to show the process of both templating techniques can be seen in Fig. 1. 


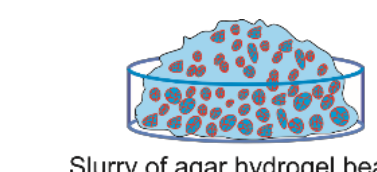

A)

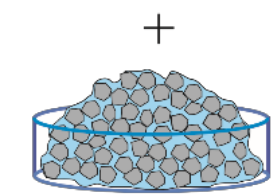

Gypsum slurry
1. Mixing slurry of agar hydrogel beads with gypsum slurry in controlled volume ratios.

\section{Hydration and} setting of the gypsum particles around the agar hydrogel bead templates.
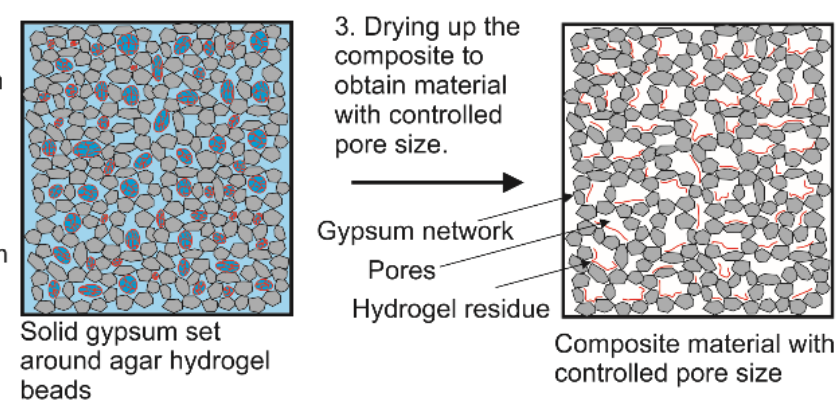

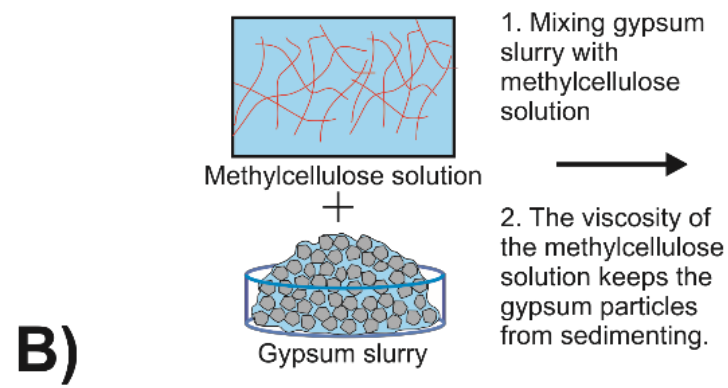

Expanded porous structure

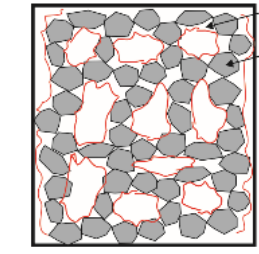

Porous gypsum composite

Gypsum network

4. Dry up the composite to obtain a material with its innate porosity expanded.

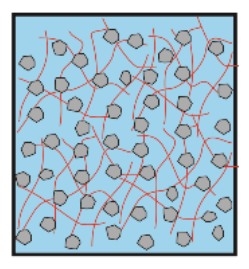

3. Hydration and setting of the gypsum particles.

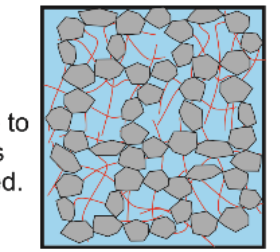

Solid gypsum-methylcellulose composite

Fig. 1. Schematics to show (A) the hydrogel beads templating technique and (B) utilising MC solution to control the porosity and pore size of gypsum composites. Gypsum slurry is mixed with either small hydrogel beads, large hydrogel beads or MC solution in controlled volume ratios. Subsequent setting of the gypsum and then drying of the composite produces materials with controlled porosity and tuneable microstructures.

\subsection{Preparation of hierarchically porous gypsum composites}

Two different methods to prepare hierarchically porous gypsum composites were used. The first involved mixing slurries of large and small hydrogel beads with controlled volume percentages, then preparing the gypsum composites as described above. The overall volume percentage of hydrogel beads in the sample was $50 \%$ and the overall volume percentage of small or large beads in their mixture was $0 \%, 10 \%, 20 \%, 30 \%, 40 \%$ or $50 \%$, with the other size hydrogel beads making up the rest of the volume. Hierarchically porous gypsum composites were also prepared by combining MC solution with hydrogel beads (small or large) and gypsum slurry in the same controlled volume ratios (overall volume percentage of templating agent was kept at 50\%). The compositions used can be found in Tables S1-3 (ESI). 


\subsection{Measurement of the composite thermal conductivity}

Before any measurements, the cartridge heater $(6.5 \mathrm{~mm}$ diameter, $60 \mathrm{~mm}$ height, purchased from RS components) was calibrated by the following procedure: A known mass of water was poured into a double jacketed calorimeter and the heater and a thermocouple (k-type, with a resolution of $0.1{ }^{\circ} \mathrm{C}$ ) were fully submerged. The calorimeter was sealed with a rubber bung which had space for the heater's wires and thermocouple to connect to a power supply and data logger (HH306A data logger thermometer, Omega), respectively. A constant current of $160 \mathrm{~mA}$ was supplied to the heater and the measured temperature change over time was used to calculate the power output as follows:

$$
Q=\frac{m C_{p} \Delta T}{t}
$$

Here $Q$ is the power output (W), $m$ is the mass of the water (g), $C_{p}$ is the specific heat capacity of water (4.184 $\mathrm{J} \mathrm{g}^{-1} \mathrm{~K}^{-1}$ ), $\Delta T$ is temperature change of the water $(\mathrm{K})$ and $t$ is time (s). This experiment was performed three times to give an average power output of $2.10 \pm 0.05 \mathrm{~W}$. For testing of the gypsum control sample and the porous gypsum composites, the sample was placed inside a cylindrical water jacket that was open at both ends (inner dimensions were 60 mm diameter and $180 \mathrm{~mm}$ length). The samples were moulded in such a way that they fit inside the water jacket and were in contact with the water-cooled $\left(15^{\circ} \mathrm{C}\right)$ glass surfaces. The heater was placed in the hole in the centre of the composites which travels the full length of the sample. Three thermocouples were placed in the sample at three different distances from the heater and to a depth of half the sample height (see Fig. 2B). Addition of insulating foam to both ends of the sample was done to minimise any axial heat loss. The heater was then switched on and the sample was heated until it reached steady state. Once steady state was achieved, the temperatures at each location could be used to calculate the thermal conductivity using the following equation[13]:

$$
\kappa=\frac{Q \ln \left(\frac{r_{x}}{r_{1}}\right)}{2 \pi L \Delta T} .
$$

Here $\kappa$ is the thermal conductivity ( $\mathrm{W} \mathrm{m}^{-1} \mathrm{~K}^{-1}$ ), $r_{1}$ is the distance between the centre of the sample and the closest thermocouple (m), $r_{x}$ is the distance between the centre of the sample and the thermocouple at location $x(\mathrm{~m}), L$ is the length of the sample (m) and $\Delta T$ is the temperature difference between the thermocouple at location 1 and the thermocouple at 
location $x$. A similar radial heat flow setup has been used elsewhere where they reported an uncertainty of less than 2\%.[14]

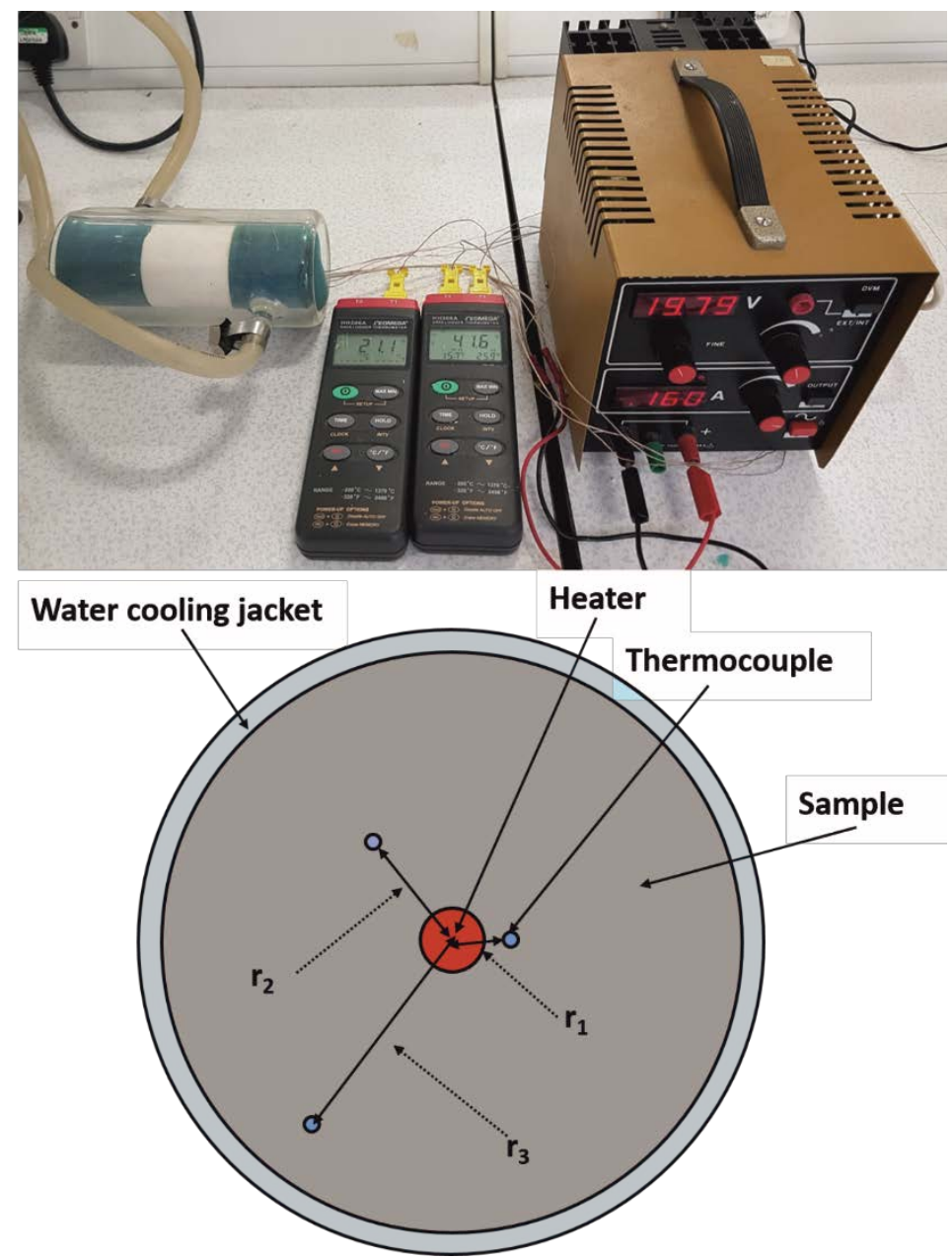

Fig. 2. The radial heat flow experimental setup for measuring the thermal conductivity of the porous composites. The schematic shows an illustration of a cross-section of a sample during a measurement.

\subsection{Mechanical properties}

Fresh samples of porous gypsum composites without holes were prepared and subjected to compression by using a Lloyds LS100 testing apparatus equipped with a $100 \mathrm{kN}$ load cell. A preload of $10 \mathrm{~N}$ was applied and the sample was compressed at a rate of $4 \mathrm{~mm} \mathrm{~min}^{-1}$. The force applied at structural failure was used to calculate the compressional strength by normalising with the cross-sectional area. The Young's modulus was determined from the gradient of the linear elastic region of the stress/strain plots. 


\subsection{Image analysis}

Hydrogel beads after blending for different amounts of time were dispersed in water and images were taken in bright field light using an Olympus BX-51 optical microscope fitted with a DP70 CCD camera. A sample size of 150 hydrogel beads at each blending time were measured and the size analysis was done using Image $\mathrm{J}$ software. Gypsum and porous gypsum composites were viewed using a Hitachi TM-1000 scanning electron microscope.

\section{Results and Discussion}

\subsection{Hydrogel bead size analysis}

In order to be able to investigate the effect of pore size on the properties of the porous gypsum composites, it was important to be able to control the size of the hydrogel beads. This was necessary, as the average pore size is dependent on the average size of the hydrogel beads used in the templating process. Average hydrogel bead size was measured after blending for different amounts of time between 10 - 600 seconds and their size distributions were obtained by analysing images of the hydrogel beads dispersed in water. The average size of the hydrogel beads varied from $600 \pm 300 \mu \mathrm{m}$ (10 seconds blending) to $100 \pm 50 \mu \mathrm{m}$ (600 seconds blending). These two size distributions of hydrogel beads were used and will be henceforth be referred to as 'large beads' and 'small beads'. Histograms showing their size distribution and optical microscopy images showing their morphology can be seen in Figs. 3A-3D. The hydrogel beads were of irregular shape so they were measured through their widest section. 

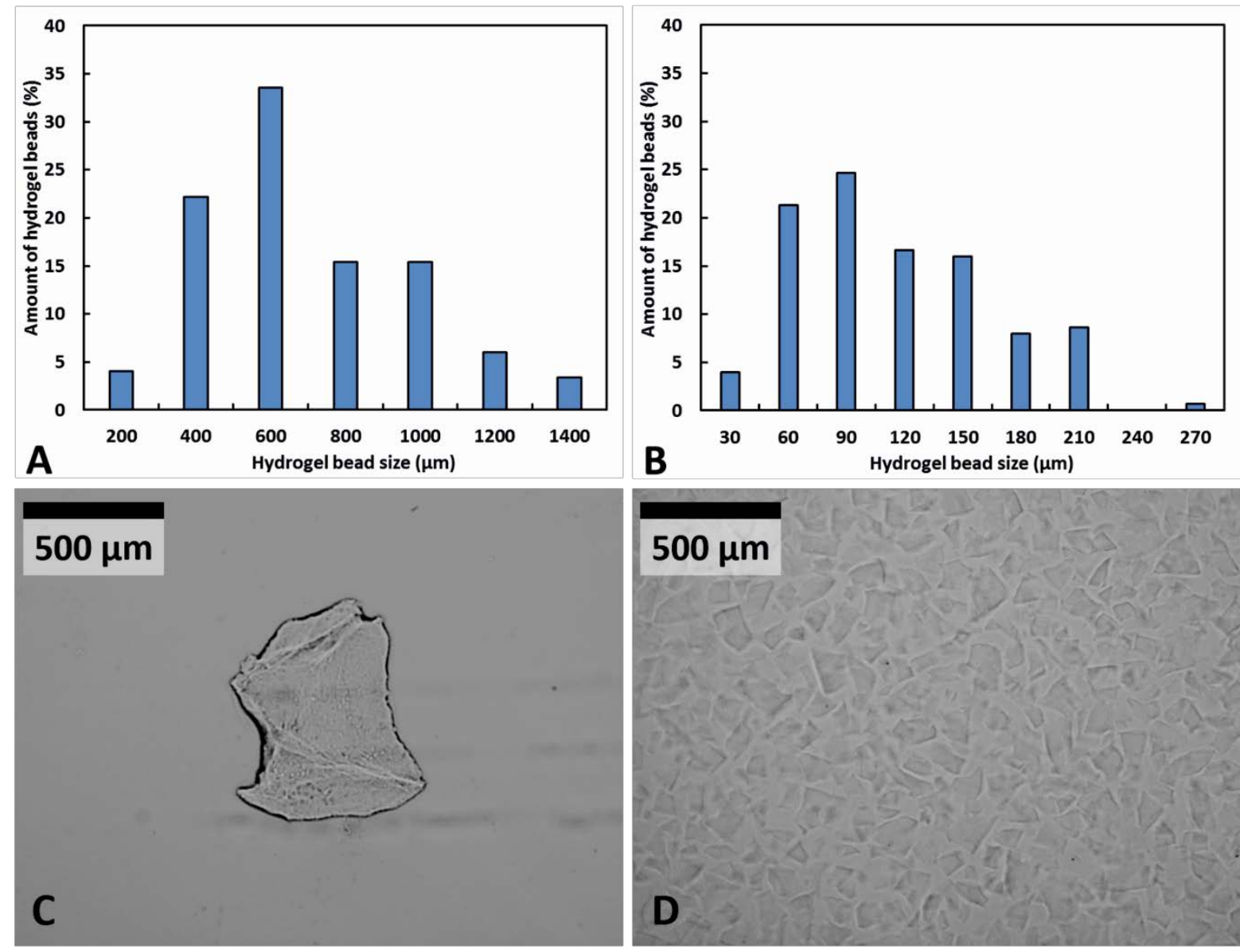

Fig. 3. (A) and (B) show size distribution diagrams produced by measuring an average of 150 large and 150 small beads, respectively. The histograms represent the percentage of the hydrogel beads of various sizes (largest dimension). (C) and (D) are optical microscopy images of a large agar bead and small agar beads dispersed in water, respectively.

\subsection{Porosity and volume reduction of the composites}

After drying of the composites, they were weighed and their dimensions were measured. Images of the samples produced using each method can be seen in Fig. S1 (ESI). Their densities and volumes were compared to the gypsum control sample to calculate the porosity and the volume reduction of the porous composites. It is worthwhile to note that gypsum plaster alone is a porous material[15], therefore the calculated porosity of the composites is in fact the reduction in density compared to gypsum plaster produced without hydrogel beads or MC solution. It was calculated as follows:

$$
\theta=\left(1-\frac{\rho}{\rho_{0}}\right) \times 100
$$


Here $\theta$ is the porosity (\%), $\rho$ is the density of porous composite $\left(\mathrm{g} \mathrm{cm}^{-3}\right), \rho_{0}$ is the density of gypsum control sample $\left(\mathrm{g} \mathrm{cm}^{-3}\right)$. The volume reduction was calculated by equation (4):

$$
V_{\text {red }}=\left(1-\frac{V}{V_{0}}\right) \times 100
$$

Here $V_{\text {red }}$ is the volume reduction (\%), $V$ is the volume of porous composite $\left(\mathrm{cm}^{3}\right), V_{0}$ is the volume of gypsum control sample $\left(\mathrm{cm}^{3}\right)$. Figs. S2A-S2C (ESI) show the porosity and volume reduction of the porous gypsum composites produced using three different methods. It was found that changing the method used to introduce porosity did not impact the volume reduction or the porosity of the samples, and that these properties were solely dependent on the volume of large agar beads, small agar beads or MC solution added. This method gives a significant amount of control over the porosity of the dried sample as the porosity is approximately the same as the volume percentage of porogenic agent used during formulation. The reductions in volume were insignificant ( $<3 \%$ ) at all the volumes of porogenic agent used, however they were still taken into account when calculating the porosity of the samples.

The use of large or small hydrogel beads to produce the porous composites works by dispersing hydrogel beads within the gypsum slurry which will then harden around the beads. Upon subsequent drying of the samples, evaporation of water from the trapped hydrogel beads will leave voids within the sample that directly reflect the size of the beads used. Note that this method works only when the surrounding matrix allows for the water to leave the system. Furthermore, the porosity is slightly lower than the volume percentage of hydrogel beads added to the gypsum slurry, possibly due to syneresis of water from the hydrogel due to the osmotic pressure mismatch upon mixing with the gypsum slurry. However, this effect is very small.

When using MC solution to control the porosity of the composites, the viscosity of this hydrocolloid solution suspends the gypsum particles during the setting process. The low solubility gypsum powder $\left(\mathrm{CaSO}_{4} \cdot 0.5 \mathrm{H}_{2} \mathrm{O}\right)$ first hydrates in solution to produce $\mathrm{CaSO}_{4} \cdot 2 \mathrm{H}_{2} \mathrm{O}$, which has an even lower solubility and so quickly recrystallizes and precipitates out in the form of needles and platelets. Interlocking of these structures forms a rigid structure[16]. As the volume of the aqueous MC solution increases, the longer it takes for the interlocking to occur and so an increased viscosity of the aqueous phase added was necessary to stop the sedimentation of gypsum particles before the gypsum composite could harden. Drying of the samples essentially leaves gypsum plaster with its (already present) porosity expanded by a controlled amount, dependent on the volume percentage of MC solution initially added. 


\subsection{Thermal conductivity of porous gypsum composites}

The total thermal conductivity $\left(\kappa_{T}\right)$ of a porous material is assumed to be due to four different contributions[17]:

$$
\kappa_{T}=\kappa_{s}+\kappa_{g}+\kappa_{c}+\kappa_{r}
$$

Here $\kappa_{s}$ is the contribution due to conduction through the solid material, $\kappa_{g}$ is the conduction through the gas within the material, $\kappa_{c}$ is the convection within the pores and $\kappa_{r}$ represents thermal radiation. In our porous composite materials, there will also be a contribution due to the presence of hydrocolloid residue present within the pores after drying. However, the amount of solid gelling agent (agar or MC) is so small (ca. $0.35-3 \%$ by mass of agar or ca. $0.09-0.75 \%$ by mass of MC) that it can be deemed insignificant. Other studies have shown that for heat transfer in closed pore materials with a pore size less than $4 \mathrm{~mm}$ and in open pore materials with a pore size less than $2 \mathrm{~mm}$, convection only plays a minor role[18,19]. Finally, thermal radiation is well known to be negligible for porous materials with relative density greater than $0.2[20]$.

We investigated how the variation of the microstructure of the porous gypsum composites affected their thermal conductivities. Three different methods were used to prepare these materials: Large hydrogel beads or small hydrogel beads as a template produced porous gypsum composites with an average pore size that reflected the size of the hydrogel beads used. In the case of gypsum composites fabricated by viscous trapping with MC solution the porous materials did not have fixed pore size as it expanded the innate porosity of the gypsum particle network during setting. Figs. 4A and 4B show the thermal conductivity of these materials and the reduction in thermal conductivity when compared to the gypsum control sample, which did not have hydrogel beads or MC solution added, respectively. 

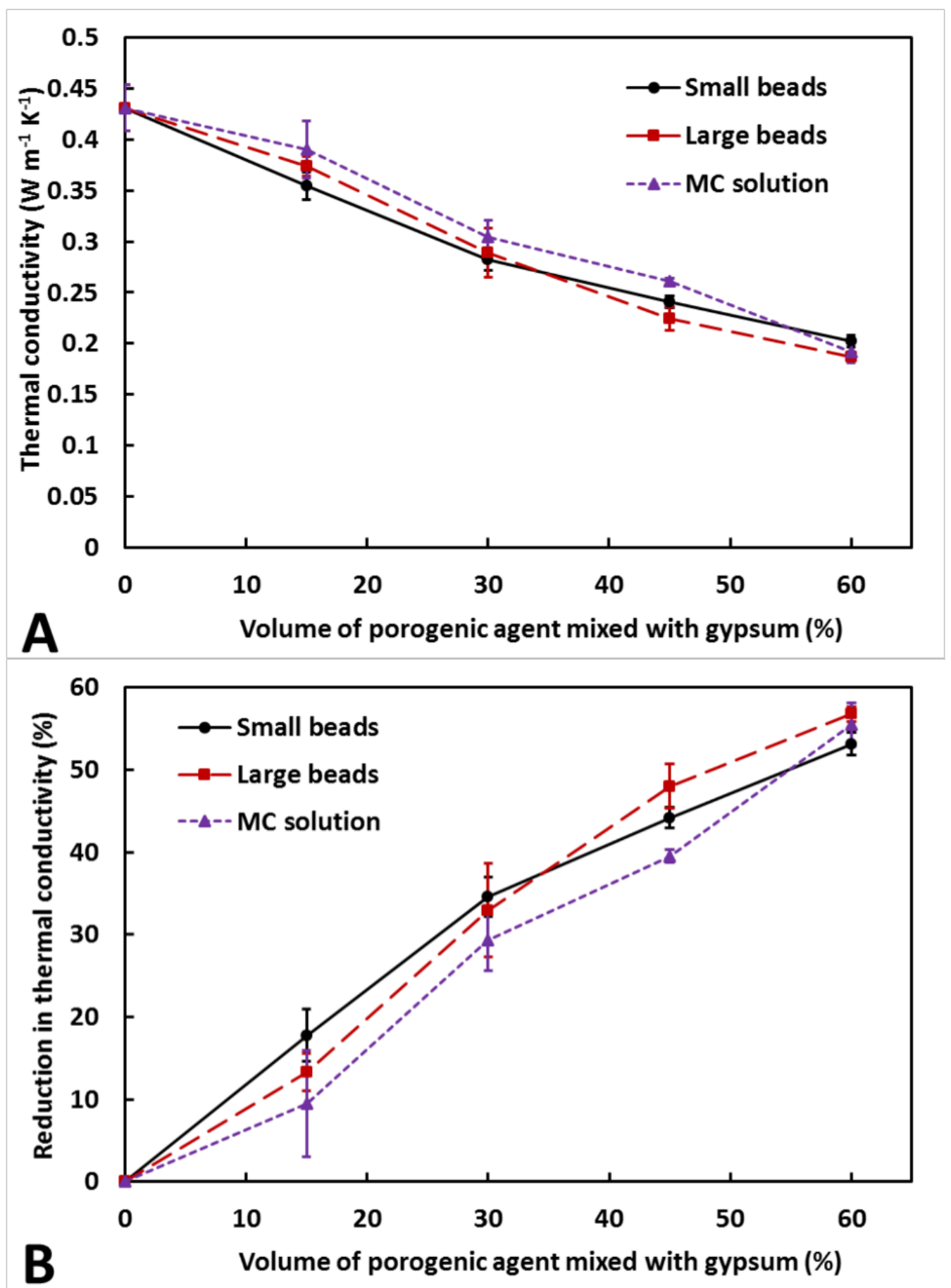

Fig. 4. (A) The thermal conductivity of the porous gypsum composites as a function of the volume of template (large beads, small beads or MC solution) initially mixed with the gypsum slurry. (B) The reduction in thermal conductivity when compared to the gypsum control sample as calculated in equation (6). Each data point is an average result of three separate samples with the error bars being the standard deviation.

The reduction in thermal conductivity was calculated as follows:

$$
\kappa_{\text {red }}=\left(1-\frac{\kappa}{\kappa_{0}}\right) \times 100
$$


Here $\kappa_{\text {red }}$ is the reduction in thermal conductivity (\%), $\kappa$ is the thermal conductivity of the porous composite $\left(\mathrm{W} \mathrm{m}^{-1} \mathrm{~K}^{-1}\right.$ ) and $\kappa_{0}$ is the thermal conductivity of the gypsum control sample $\left(\mathrm{W} \mathrm{m}^{-1} \mathrm{~K}^{-1}\right)$. The measured thermal conductivity for gypsum plaster alone was $0.43 \pm 0.02 \mathrm{~W}$ $\mathrm{m}^{-1} \mathrm{~K}^{-1}$ which is in within the range of other results found in the literature[2]. The thermal conductivities of the porous composites were found to decrease linearly as the volume percentages of template used during formulation were increased. Note that the reduction in thermal conductivity closely reflects the initial volume percentage of template used to prepare the composites i.e. when using $30 \%$ by volume of template during the formulation process, the composites show a reduction in the thermal conductivity by approximately $30 \%$.

Fitting a simple linear equation to each data set in Fig. 4B allows for a reasonable approximation of the reduction in thermal conductivity when adding different volume percentages of template. These are shown in equations (7)-(9) for composites prepared by using small beads, large beads, and MC aqueous solution, respectively.

$$
\begin{aligned}
\kappa_{\text {red }}=0.9599 V_{S B}, & & (\text { small beads }) \\
\kappa_{\text {red }}=1.0004 V_{L B}, & & (\text { large beads }) \\
\kappa_{\text {red }}=0.9080 V_{M C} . & & (\text { MC solution })
\end{aligned}
$$

Here $\kappa_{\text {red }}$ is the reduction in thermal conductivity (\%) and $V$ is the volume of template used to make the porous gypsum composite $(\%)$; $\mathrm{SB}=$ small beads, $\mathrm{LB}=$ large beads, $\mathrm{MC}=$ methylcellulose solution). The $\mathrm{R}^{2}$ values were $0.964,0.986$ and 0.988 for the linear fits shown in equations (7), (8) and (9), respectively. Further work would be needed to investigate whether these equations are applicable to other porous materials produced with these templating methods. Furthermore, we found that the pore size distribution within the porous gypsum composites had no impact on the thermal conductivity when compared at the same overall porosity.

One possible way to reduce the thermal conductivity of a porous composite material at a fixed porosity could also involve the reduction of the thermal conductivity of the gas within the pores. This can be done in two ways: (i) by replacing air with a gas of lower thermal conductivity followed by sealing of the porous composite, or (ii) by reducing the thermal conductivity of air[21]. The Knudsen effect describes the reduction of the thermal conductivity of a gas within a pore when the pore size is comparable to or smaller than the mean free path of the gas. For air at room temperature, this is approximately $70 \mathrm{~nm}$ [22]. The Knudsen equation describes how variation of the pore size affects the thermal conductivity of the air within the pores[23]: 


$$
\kappa_{g}^{\prime}=\frac{\kappa_{g 0}^{\prime}}{\left(1+\beta\left(\frac{l_{g}}{\phi}\right)\right)}
$$

Here $\kappa_{g}^{\prime}$ is the thermal conductivity of air within the pores, $\kappa_{g 0}^{\prime}$ is the thermal conductivity of free air $\left(0.026 \mathrm{~W} \mathrm{~m}^{-1} \mathrm{~K}^{-1}\right), \beta$ is a parameter that takes into account the transfer of energy when air molecules collide with a pore boundary $(\sim 2), l_{g}$ is the mean free path of air and $\phi$ is the average pore diameter.

One can explain the lack of effect of the pore sizes on the thermal conductivity from estimates using equation (10). It can be seen that the difference in the pore sizes used to prepare our porous composites was not large enough to see any significant difference. Reduction of the pore size to $1 \mu \mathrm{m}$ or $0.1 \mu \mathrm{m}$ would be expected to show a reduction in the thermal conductivity of the air within the pores of $12 \%$ or $58 \%$, respectively. This would in turn reduce the thermal conductivity of the porous composite material. However, this would require using submicron size pores which are not achievable by using hydrogel bead templating on this length scale.

\subsection{Mechanical properties}

Through the use of three different templating techniques, porous composite gypsum materials with three very different microstructures have been obtained. We investigated how the microstructure of the porous gypsum composites affects their mechanical properties. Fresh samples without the holes for thermal conductivity testing were prepared and dried to a constant weight. They were subjected to compression until structural failure which allowed calculation of the compressional strength and Young's modulus. The results are presented in Figs. 5A-5B. It was found that increasing the size of the hydrogel bead templates used to introduce porosity from $100 \mu \mathrm{m}$ to $600 \mu \mathrm{m}$ decreased the compressional strength by approximately $50 \%$ when the overall porosity was between $15-45 \%$. When the porosity reached $60 \%$, the compressional strength was independent of the material's microstructure. Likewise, when increasing the template hydrogel bead size, the Young's modulus decreased by approximately $60 \%$ for porosity in the range $15-45 \%$. When the porosity reached $60 \%$, however, the average Young's modulus of the porous gypsum composites produced with large hydrogel beads as templates was $80 \%$ lower than the ones produced with small hydrogel beads. 

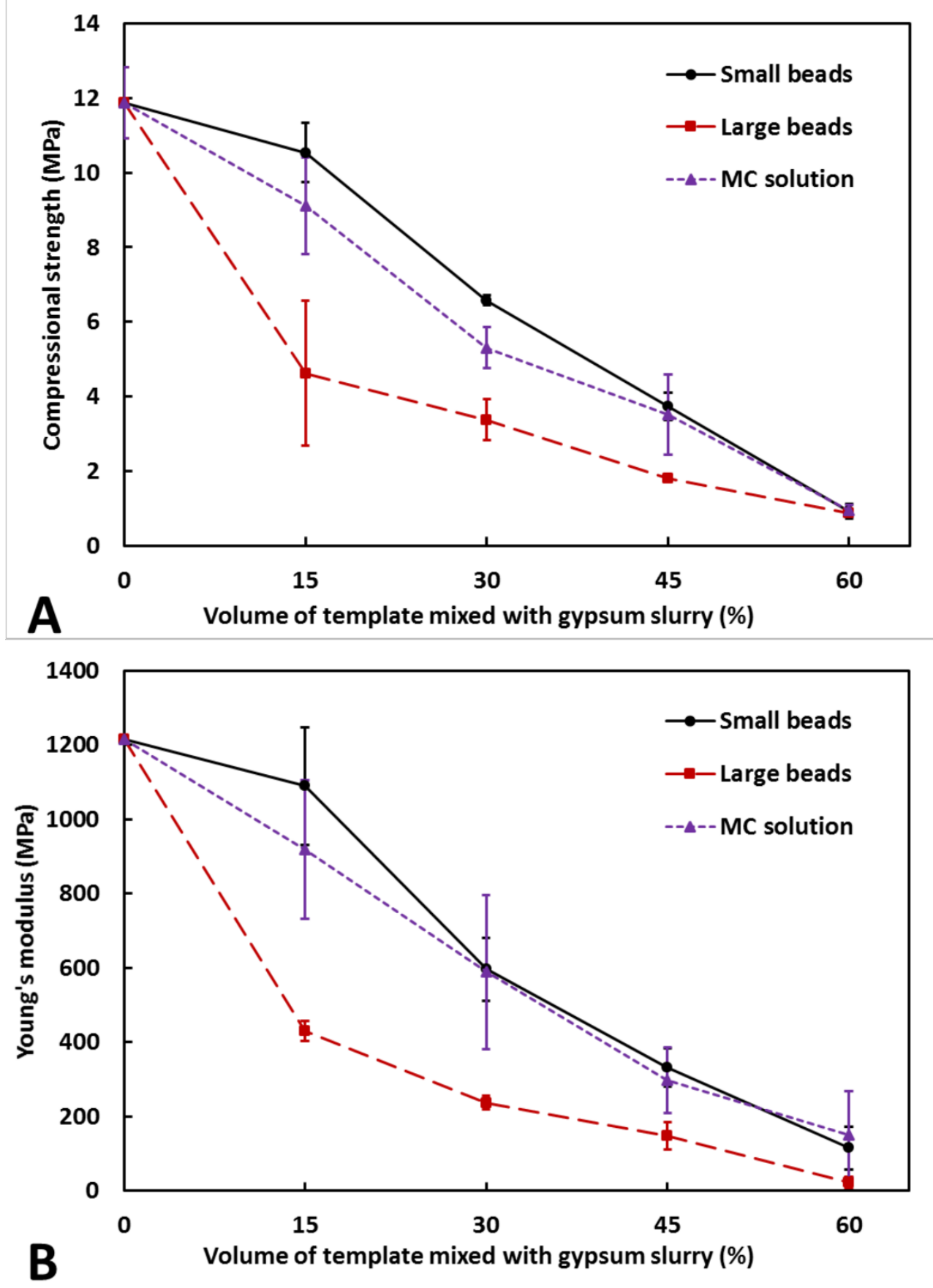

Fig. 5. (A) The compressional strength and (B) the Young's modulus of the porous gypsum composites as a function of the volume of template (small beads, large beads or MC solution) initially mixed with the gypsum slurry. Each sample was measured at least twice and the average value is shown.

The gypsum composites formulated using MC solution had compressional strengths and Young's moduli comparable to the ones produced with small beads. When using either small beads or MC solution to produce the porous gypsum composites, there was a relatively linear decrease of the compressional strength and Young's modulus with the volume percentage of 
template hydrogel of MC solution used. On the other hand, when using large beads as a template, there was a large initial decrease of approximately $60 \%$ in both the compressional strength and Young's modulus when the porosity was increased from 0 to just $15 \%$. At porosities higher than 15\% however, a linear decrease was observed. As the thermal conductivity does not vary with pore size on the length scales investigated here, but the mechanical properties do, it is possible to have materials with constant thermal conductivity and controlled mechanical properties by varying the pore size.

The phenomenon of smaller pores producing materials with enhanced mechanical properties has also been reported by other authors[24,25] and attributed to a combination of an increased surface area to volume ratio, sharing of the load between more and smaller pores and a decrease of the moment upon the walls of the small pores[25,26].

We then investigated how varying the ratio of large pores to small pores, at constant porosity, affects the mechanical properties of the porous composites. With the overall volume of hydrogel beads in the sample kept at a constant $50 \%$, the ratio of large beads to small beads was varied to produce hierarchically porous gypsum composites with controlled pore sizes and controlled ratio of large pores to small pores. Their mechanical properties are presented in Figs. S3A-S3B. The mechanical properties of the hierarchically porous composite materials increase with an increase in the overall volume of small hydrogel beads. This can be explained by Figs. $5 \mathrm{~A}-5 \mathrm{~B}$, where the composites produced using small beads had increased mechanical properties when compared to those produced using large beads.

Finally, we investigated how varying the ratio of hydrogel beads (large or small) to MC solution, at constant overall volume percentage of template, affected the mechanical properties of the hierarchically porous composites produced. The overall volume percentage of gypsum slurry used during production was kept at 50\%, while the volume percentages of MC solution and hydrogel beads were varied. The results are shown in Figs. 6A-6B. In Fig. 6A, an increase in the compressional strength can be seen when increasing the volume percentage of small hydrogel beads, with a maximum compressional strength when the volume percentages of small beads and MC solution are 30\% and 20\%, respectively. It has been suggested previously that hierarchically porous materials could have an optimum balance between different sized pores to produce materials with enhanced mechanical properties[27]. In Fig. 6B, it can be seen that as the volume percentage of large beads is increased compared to MC solution, the Young's modulus decreases. This shows there is scope for tuning material's mechanical properties at constant porosity by varying the microstructure. 

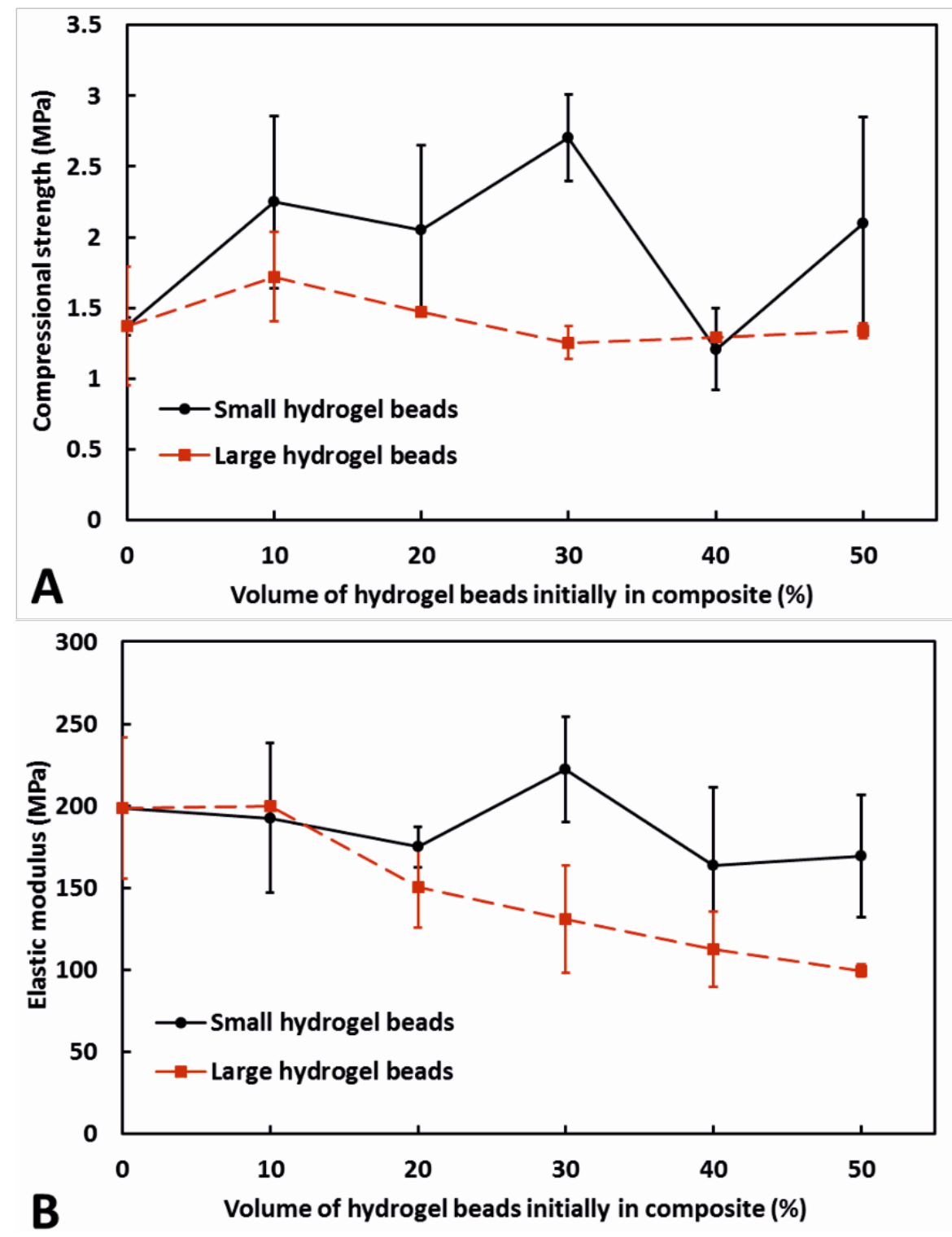

Fig. 6. Compressional strength (A) and Young's modulus (B) of hierarchically porous gypsum composites produced through a combined approach of viscous trapping with MC solution and hydrogel bead templating. The overall volume percentage of gypsum slurry was kept constant at $50 \%$. The volume percentage of hydrogel beads is shown on the $\mathrm{x}$-axis and volume percentage of MC solution is what makes up the rest i.e. when $10 \%$ by volume of hydrogel beads are used, $40 \%$ by volume of MC solution is used. Each data point is the average of at least two measurements and the error bars are the standard deviation.

\subsection{Microstructure analysis}

Samples were taken from the gypsum control sample and the porous gypsum composites produced with $60 \%$ by volume of the templates and viewed with a benchtop SEM, without a conductive coating. They were viewed at reasonable magnifications to observe the differences 
in the composite microstructures due to the presence of the different templates during the formulation process.

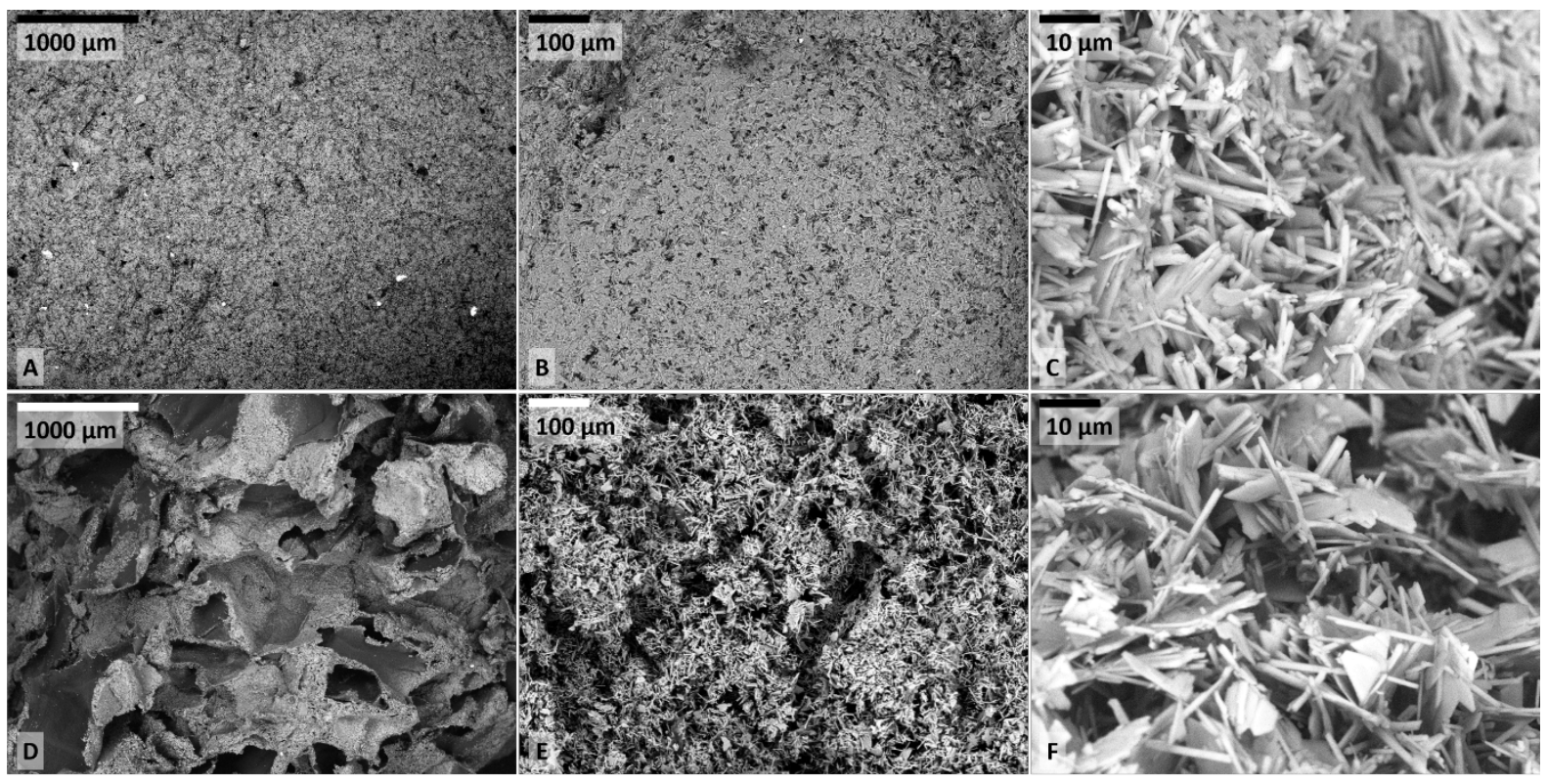

Fig. 7. (A) - (C) SEM images of the gypsum control sample at three different magnifications. The scale bars are $1000 \mu \mathrm{m}, 100 \mu \mathrm{m}$ and $10 \mu \mathrm{m}$, respectively. (D) - (F) SEM images of the porous gypsum composites produced with $60 \%$ by volume of large beads, small beads or MC aqueous solution, respectively. The magnification and scale bars are the same as the image above them.

Figs. 7A-7C show the gypsum control sample at three different magnifications whilst Figs. 7D$7 \mathrm{~F}$ show the porous gypsum composites produced with $60 \%$ by volume of large beads, small beads and MC solution, respectively. In Figs. 7A-7C one can see the innate porosity of the gypsum control samples, whilst Figs. 7D-7F show the increased porosity due to the incorporation of templating materials (hydrogel beads, MC solution) during preparation. In Fig. 7D, it can be seen that the pores present within the composites produced using large hydrogel beads are of a similar size to the original beads used before their embedding in the gypsum slurry and further drying. Furthermore, it can be observed that upon drying of the composites, the hydrogel residues stay mostly within the sample pores. Again, when using small beads as a template, the pore sizes reflect the size of the beads used, however no visible residues of hydrogel material can be seen within the pores. The larger surface area-to-volume ratio of the small hydrogel beads means that the residue produced upon drying will be much thinner so it could have intercalated in between the surrounding microcrystals among the gypsum network. Figs. 8A-8D show higher magnifications of the inner pore structures of the composites produced with large beads, small beads, 30\% MC and 60\% MC by volume, respectively. 


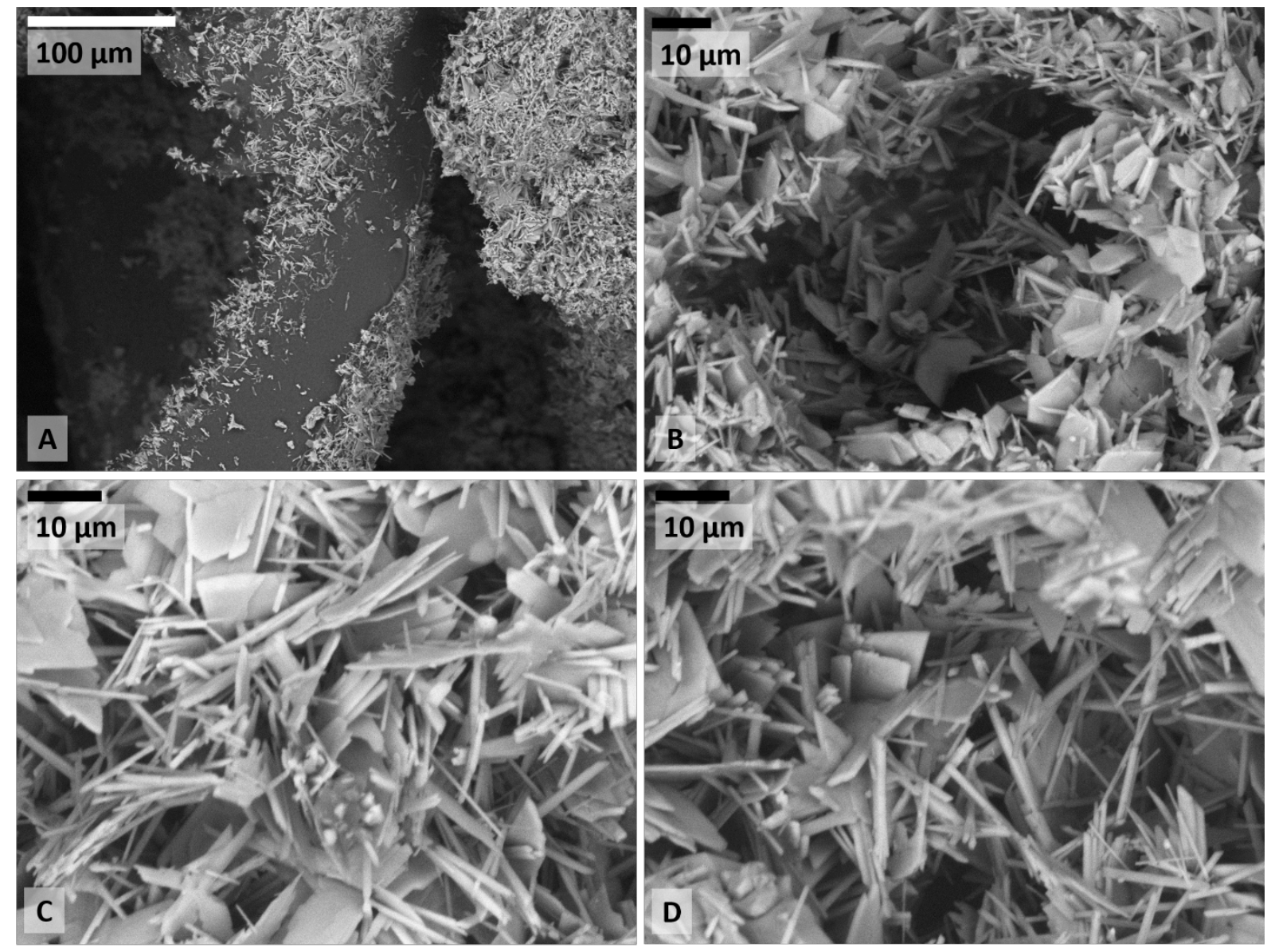

Fig. 8. The inner pore structures of composites produced using large beads (A) or small beads (B). Note that when using large beads as porosity inducing templates, the gypsum needles and platelets attach to folded film of the hydrogel residue upon drying whereas when using small beads, this is not seen. (C) and (D) show the microstructure of porous gypsum composites produced with $30 \%$ and $60 \%$ by volume MC solution, respectively.

Figs. 8A-B show the significantly different microstructures of two composites produced using hydrogel bead slurries with different size distributions. In Fig. 8A, there is evidence of the gypsum crystallite needles and platelets being attached to the hydrogel residues which means that they are separate from the continuous gypsum network. This could possibly contribute to the reduction in mechanical properties when compared to the composites produced using small beads or MC solution, as these structures are not seen within the pores of those samples. The use of MC solution produced porous composites with an expanded microstructure, as seen through comparison of Figs. 7C and 7F and 8C-8D. With the expansion of the gypsum network, the mechanical properties decreased. Similarly to the gypsum composites produced using small hydrogel beads, we did not observe any hydrogel residue. These differences in the internal pore 
structures could explain the varying mechanical properties at the same porosity. The composites produced with large hydrogel beads were weaker possibly due to the presence of hydrogel residues within the pores. The needles and platelets attached to the residues will not be contributing to the mechanical properties of the composites, as they are not part of the continuous gypsum network.

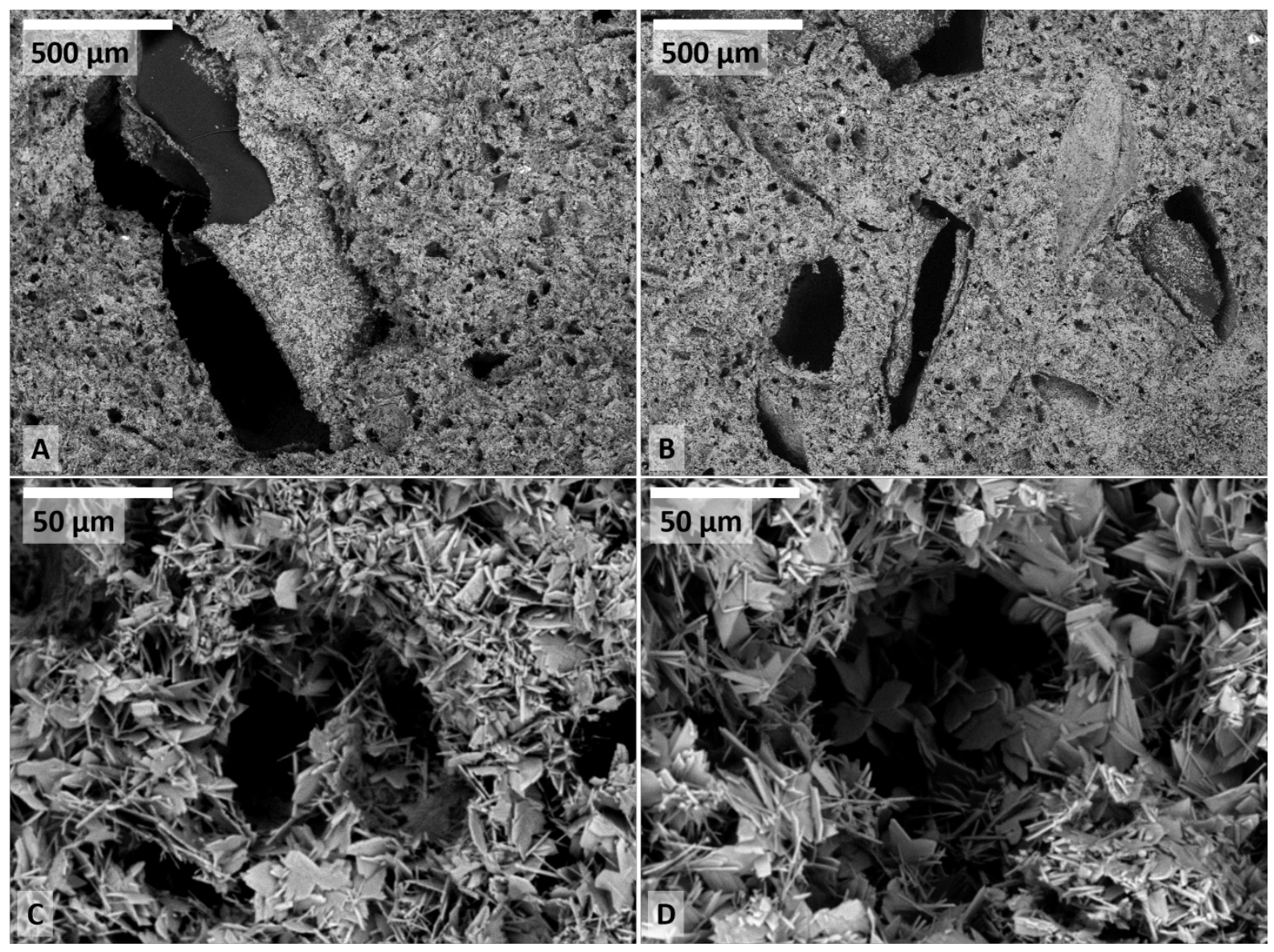

Fig. 9. SEM images of hierarchically porous gypsum composites produced using a dual size hydrogel bead templating process (A) and (B) or a combination of small hydrogel beads and MC solution (C) and (D). (A) has a porosity of $50 \%$ that was induced by $30 \%$ large beads and $20 \%$ small beads whereas (B) has the same porosity but it was induced by $20 \%$ large beads and $30 \%$ small beads. The sample seen in (C) has a porosity of $50 \%$ from 10\% MC solution and 40\% small beads and (D) shows a sample produced with 10\% small beads and 40\% MC solution.

Hierarchically porous gypsum composites produced using two different sized hydrogel beads or hydrogel beads and MC solution have also been visualised. The two distinct pore sizes produced as a result of different sized hydrogel beads used can be easily seen and is demonstrated in Figs. 9A-9B. In Figs. 9A-9B, one can observe the hierarchical porosity present in the gypsum composites. Moreover, hydrogel residues are again present within the large pores, with gypsum needles and platelets attached to them. The hierarchically porous gypsum 
composites fabricated through the use of viscous trapping with MC solution and hydrogel templating with different sized beads are shown in Figs. 9C-9D. It was possible to observe evidence of both mechanisms of controlling the porosity. The difference in how closely packed the gypsum particles are packed can be seen in Figs. 9C-9D. With an increase in the volume percentage of MC solution used to produce the porous composites, the needles and platelets are less tightly packed. Combining these methods to produce hierarchically porous materials gives scope for the preparation of a range of materials with well-defined, controllable microstructures.

\section{Conclusions}

A hydrogel bead templating technique has been used to produce porous gypsum composites with a controllable porosity, pore size and microstructure. Preparation of agar hydrogel beads of two different size distributions was achieved by blending agar hydrogel for different amounts of time. These were then used as templates to produce hierarchically porous materials with pore sizes that reflect the sizes of the hydrogel beads used. An alternative viscous trapping method to produce porous gypsum composites has been developed which utilises a viscous MC aqueous solution to suspend gypsum particles during the setting process. This produced gypsum composites with an expanded innate porosity without a controlled pore size, but with a porosity directly reflected by the volume percentage of MC solution used during the composite formulation. These methods allowed us to introduce porosities of up to $60 \%$ with negligible volume reductions.

Investigations into how the porosity and microstructure of the composites affected their thermal conductivities were performed. Increasing their porosity showed a linear decrease in their thermal conductivities. This decrease in thermal conductivity could be well controlled by using different volume percentages of templating agent i.e. to obtain a reduction in thermal conductivity of $30 \%, 30 \%$ by volume of templating agent would be used during formulation. This allowed for linear equations for the prediction of reduction in thermal conductivity to be obtained. Furthermore, it was found that at the pore sizes and porosities studied here, the thermal conductivity was independent of the material microstructure and the hierarchy of the pores. On the other hand, the Young's modulus and compressional strength were strongly dependent on the microstructure of the gypsum composites. Large decreases in the mechanical properties of the composites produced using large hydrogel beads was seen when compared to 
the samples produced by templating small hydrogel beads or MC solution at the same porosity. We attribute this decrease in mechanical properties when using large hydrogel beads as templates to the fact that they leave residues upon drying. Gypsum needles and platelets attach to the residue and therefore do not contribute to the overall mechanical properties of the porous composite. SEM imaging of the samples revealed different microstructures of the composites produced for the three templates studied. It was clearly seen that the pore size was dependent on the size of the hydrogel beads used during the sample preparation. An expansion of the gypsum network due to a smaller amount of gypsum particles being suspended in the MC solution in same overall volume during the setting process was also seen. The described methodology can produce materials with a controllable thermal conductivity by varying the porosity of the composites, followed by tuning the mechanical properties by changing the pore size at a constant porosity.

\section{Acknowledgements}

B.T. acknowledges the financial support from EPSRC/Unilever CASE studentship for his PhD study.

\section{References}

[1] Y.W.I. Rahmanian, Thermal conductivity of gypsum at high temperatures: a combined experimental and numerical approach, Acta Polytech. 49 (1) (2009) 16-20.

[2] G. Thomas, Thermal properties of gypsum plasterboard at high temperatures, Fire Mater. 26 (1) (2002) 37-45, https://doi.org/10.1002/fam.786.

[3] D.S. Smith, A. Alzina, J. Bourret, B. Nait-Ali, F. Pennec, N. Tessier-Doyen, K. Otsu, H. Matsubara, P. Elser, U.T. Gonzenbach, Thermal conductivity of porous materials, J. Mater. Res. 28 (17) (2013) 2260-2272, https://doi.org/10.1557/jmr.2013.179.

[4] H. Li, Q. Zeng, S. Xu, Effect of pore shape on the thermal conductivity of partially saturated cement-based porous composites, Cem. Concr. Compos. 81 (2017) 87-96, https://doi.org/10.1016/j.cemconcomp.2017.05.002.

[5] N. Flores Medina, M.M. Barbero-Barrera, Mechanical and physical enhancement of gypsum composites through a synergic work of polypropylene fiber and recycled isostatic graphite filler, Constr. Build. Mater. 131 (2017) 165-177, https://doi.org/10.1016/j.conbuildmat.2016.11.073.

[6] V. Rheinheimer, Y. Wu, T. Wu, K. Celik, J. Wang, L. De Lorenzis, P. Wriggers, M.-H. Zhang, P.J.M. Monteiro, Multi-scale study of high-strength low-thermal-conductivity cement composites containing cenospheres, Cem. Concr. Compos. 80 (2017) 91-103, https://doi.org/10.1016/j.cemconcomp.2017.03.002. 
[7] S. Song, Y. Zhang, Construction of 3D multiple networks skeleton by Thiol-Michael addition click reaction to fabricate novel polymer/graphene aerogels with exceptional thermal conductivity and mechanical properties, J. Mater. Chem. A. (2017), accepted manuscript, https://doi.org/10.1039/C7TA07173F.

[8] P. Jana, V. Fierro, A. Pizzi, A. Celzard, Thermal conductivity improvement of composite carbon foams based on tannin-based disordered carbon matrix and graphite fillers, Mater. Des. 83 (2015) 635-643, https://doi.org/10.1016/j.matdes.2015.06.057.

[9] S. Gbewonyo, A.W. Carpenter, C.B. Gause, N.R. Mucha, L. Zhang, Low thermal conductivity carbon fibrous composite nanomaterial enabled by multi-scale porous structure, Mater. Des. 134 (2017) 218-225, https://doi.org/10.1016/j.matdes.2017.08.050.

[10] J.K. Carson, S.J. Lovatt, D.J. Tanner, A.C. Cleland, Thermal conductivity bounds for isotropic, porous materials, Int. J. Heat Mass Transfer 48 (11) (2005) 2150-2158, https://doi.org/10.1016/j.ijheatmasstransfer.2004.12.032.

[11] M. Rutkevičius, S.K. Munusami, Z. Watson, A.D. Field, M. Salt, S.D. Stoyanov, J. Petkov, G.H. Mehl, V.N. Paunov, Fabrication of novel lightweight composites by a hydrogel templating technique, Mater. Res. Bull. 47 (4) (2012) 980-986, https://doi.org/10.1016/j.materresbull.2012.01.014.

[12] K.C. Labropoulos, D.E. Niesz, S.C. Danforth, P.G. Kevrekidis, Dynamic rheology of agar gels: theory and experiments. Part I. Development of a rheological model, Carbohydr. Polym. 50 (4) (2002) 393-406, https://doi.org/10.1016/S0144-8617(02)00084-X.

[13] T.M. Tritt, D. Weston, Measurement techniques and considerations for determining thermal conductivity of bulk materials, in: T.M. Tritt (Ed.), Therm. Conduct. Theory, Prop. Appl., Springer US, Boston, MA, 2004: pp. 187-203, https://doi.org/10.1007/0-387-26017-X_8.

[14] D.R. Flynn, A radial-flow apparatus for determining the thermal conductivity of loose-fill insulations to high temperatures, J. Res. Nat. Bur. Stand., Sect. C 67 (1963) 129-137.

[15] A.J. Lewry, J. Williamson, The setting of gypsum plaster - Part II The development of microstructure and strength, J. Mater. Sci. 29 (21) (1994) 5524-5528, https://doi.org/10.1007/BF00349943.

[16] A.J. Lewry, J. Williamson, The setting of gypsum plaster - Part I The hydration of calcium sulphate hemihydrate, J. Mater. Sci. 29 (20) (1994) 5279-5284, https://doi.org/10.1007/BF01171536.

[17] B. Notario, J. Pinto, M.A. Rodríguez-Pérez, Nanoporous polymeric materials: A new class of materials with enhanced properties, Prog. Mater. Sci. 78 (2016) 93-139, https://doi.org/10.1016/j.pmatsci.2016.02.002.

[18] D.K. Hale, The physical properties of composite materials, J. Mater. Sci. 11 (11) (1976) 21052141, https://doi.org/10.1007/BF02403361.

[19] M. Alvarez-Lainez, M.A. Rodríguez-Pérez, J.A. de Saja, Thermal conductivity of open-cell polyolefin foams, J. Polym. Sci. Part B Polym. Phys. 46 (2) (2008) 212-221, https://doi.org/10.1002/polb.21358.

[20] E. Solórzano, M.A. Rodríguez-Pérez, J. Lázaro, J.A. de Saja, Influence of Solid Phase Conductivity and Cellular Structure on the Heat Transfer Mechanisms of Cellular Materials: Diverse Case Studies, Adv. Eng. Mater. 11 (10) (2009) 818-824, https://doi.org/10.1002/adem.200900138. 
[21] B. Notario, J. Pinto, E. Solórzano, J.A. de Saja, M. Dumon, M.A. Rodríguez-Pérez, Experimental validation of the Knudsen effect in nanocellular polymeric foams, Polym. (United Kingdom). 56 (2015) 57-67, https://doi.org/10.1016/j.polymer.2014.10.006.

[22] S.. Jennings, The mean free path in air, J. Aerosol Sci. 19 (2) (1988) 159-166, https://doi.org/10.1016/0021-8502(88)90219-4.

[23] X. Lu, R. Caps, J. Fricke, C.T. Alviso, R.W. Pekala, Correlation between structure and thermal conductivity of organic aerogels, J. Non. Cryst. Solids. 188 (3) (1995) 226-234, https://doi.org/10.1016/0022-3093(95)00191-3.

[24] M. Rutkevičius, Z. Austin, B. Chalk, G.H. Mehl, Q. Qin, P.A. Rubini, S.D. Stoyanov, V.N. Paunov, Sound absorption of porous cement composites: effects of the porosity and the pore size, J. Mater. Sci. 50 (9) (2015) 3495-3503, https://doi.org/10.1007/s10853-015-8912-5.

[25] Z. Li, L. Yang, Y. Li, Y. Yang, C. Zhou, Y. Ding, J. Zhao, Y. Li, Effects of pore size on the mechanical properties of three-dimensionally ordered macroporous nickel, Mater. Des. 45 (2013) 52-55, https://doi.org/10.1016/j.matdes.2012.09.009.

[26] Z.G. Xu, J.W. Fu, T.J. Luo, Y.S. Yang, Effects of cell size on quasi-static compressive properties of Mg alloy foams, Mater. Des. 34 (2012) 40-44, https://doi.org/10.1016/j.matdes.2011.07.066.

[27] L.L.C. Wong, P.M. Baiz Villafranca, A. Menner, A. Bismarck, Hierarchical polymerized high internal phase emulsions synthesized from surfactant-stabilized emulsion templates, Langmuir. 29 (20) (2013) 5952-5961, https://doi.org/10.1021/la3047643. 